\title{
Immunopathology of lung diseases: introduction for the special issue
}

\author{
Tracy Hussell ${ }^{1}$ • Aleksander M. Grabiec ${ }^{1}$
}

Received: 8 May 2016 / Accepted: 9 May 2016/Published online: 19 May 2016

(C) Springer-Verlag Berlin Heidelberg 2016

This issue of Seminars in Immunopathology focuses on lung innate immunity and the recently identified mechanisms contributing to this unique environment. It was in the 1980s that T-cell subsets were first described by the cytokines that induced them and the cytokines they then produced [1,2]. This segregation allowed more flexibility in the immune system, fine-tuning responses to eliminate very different antigens or pathogens. Sub-division to define optimal function, however, has now expanded to natural killer (NK) cells, innate lymphoid cells (ILCs), dendritic cells (DCs) and macrophages. Though polarising conditions in vitro provide clarity in our understanding of influencing forces, they do not tell us how this occurs in vivo, how it is regulated, or whether it contributes to common pathological conditions.

The discovery of tissue-specific regulation of innate and adaptive immunity has transformed our understanding of the immune system, provided clarity for otherwise unexplainable phenomena, and opened up a new area for scientific discovery [3]. Immunity adapts to tissue-specific cues that in turn maintain or restore homeostatic physiology. Forces driving the tissue adaptation in disease alter immune phenotype and reactivity, which can have deleterious consequences. The lung is a prime example of site-specific forces dictating the balance between health and disease. In this issue we examine lung

This is the introduction for Immunopathology of Lung Diseases - Drs Tracy Hussell and Aleksander M. Grabiec

Tracy Hussell

tracy.hussell@manchester.ac.uk

1 Manchester Collaborative Centre for Inflammation Research, Core Technology Facility, The University of Manchester, 46 Grafton Street, M13 9NT Manchester, UK innate immunity in this precise tissue-specific context, the impact of ILCs and neutrophils on health and disease, macrophage and DC adaptation and the consequences of disturbances in chronic lung disease and infection.

To some extent, tissue-specific influences on innate immunity should depend on what the tissue requires of that innate immune cell. Alveolar macrophages reside in a prime location for interaction with environmental and commensal microorganisms. Their role appears to be to sift the harmless from the dangerous and also perform domestic duties, keeping airspace clutter within tolerable margins [4]. These duties include removal of surfactant proteins, cellular and matrix debris, and apoptotic cells. Cell turnover by apoptosis is necessary, and removal of apoptotic bodies is essential to prevent inflammation-inducing secondary necrosis. In this issue, Grabiec and Hussell detail the mechanisms and impact of apoptotic cell uptake (efferocytosis) on airway macrophage function. Clearly, clearance of self-cells or proteins must be performed without activating the macrophage, otherwise peripheral tolerance will be overcome, leading to chronic inflammation or autoimmunity. The process of efferocytosis therefore triggers anti-inflammatory cascades. On the other hand, clearance of pulmonary pathogens by airway macrophages may require assistance from other cells, which necessitates macrophage production of chemokines that culminates in inflammatory cell recruitment. Eventually these recruited cells will themselves undergo apoptosis and require efferocytosis. Here we have a conundrum where two opposing functions are requested of airway macrophages: efferocytosis and inflammation. Writing in this issue, Robb and colleagues discuss the impact of neutrophil and eosinophil apoptosis and their clearance from the airways on the resolution of the inflammatory response.

Despite the considerable advances made in recent years in our understanding of airway macrophage origin, heterogeneity, longevity and turnover, there are still many unanswered questions [5]. Do original airway macrophages perform 
housekeeping, inflammatory and repair roles? Are these functions catered for by different macrophage populations, e.g. resident versus recruited? Does function actually drive macrophages down specific developmental paths? For example, efferocytosis of apoptotic cells or engulfment of matrix products may induce a wound-healing macrophage phenotype; function driving form. DCs have also been extensively subdivided based on their origin and on the mechanisms by which they are activated and which they may use to coordinate downstream inflammatory responses in the lung (reviewed in this issue by Cook and MacDonald). However, innate immune cell polarisation may also be driven by cellular connections within the local environment. Location is likely to be of considerable importance in determining cell sub-type dominance, as required functions will be very different at different sites. Consider macrophages or DCs in lymph nodes versus those in the tissue or mucosal lumen, for example [6]. In this issue, Bhattacharya and Westphalen discuss the influence of the bronchial epithelium on airway macrophage function in homeostasis and the importance of this interaction in orchestrating the macrophage-driven immune response.

Balanced interplay between innate and adaptive immune responses is required for timely removal of pathogenic microorganisms infecting the lung, resolution of the inflammatory process and repair of the damaged tissue. Respiratory viral infections are the most prominent example of how the balance between pathogen elimination and prevention of immunemediated lung injury is achieved [7]. Understanding the immune mechanisms underlying an effective, but self-limiting anti-viral response is particularly important in the context of the emergence of novel respiratory viruses, such as SARS and MERS coronaviruses, and the constant threat posed by pandemic influenza. Recent advances in this field are reviewed in this pages by Newton and colleagues.

Allergic lung inflammation and asthma are generally associated with uncontrolled $\mathrm{T}$ helper 2-cell-mediated inflammation caused by the adaptive immune response to airway allergens. However, the recent discovery of ILCs, a novel innate cell population, has greatly improved our understanding of the importance of innate immunity in these conditions [8]. Van Rijt and colleagues discuss the involvement of type 2 ILCs in allergic asthma, in particular their role as an early innate source of type 2 cytokines, such as interleukin (IL)-13 and IL-5, which are main drivers of allergic inflammation. Disruption of both innate and adaptive immune responses also contributes to chronic obstructive pulmonary disease (COPD), which is among the leading causes of morbidity and mortality worldwide [9]. Also in this issue, Caramori and colleagues detail the roles of innate and adaptive immune cell populations in COPD immunopathology in its stable phase and during exacerbations. The authors also highlight the contributions of altered cytokine networks to chronic inflammation and airway remodelling in COPD.
Uncontrolled production of inflammatory cytokines, predominantly by innate immune cells and resident cells of the respiratory tract, plays a key role in driving airway damage not only in COPD, but also in many other lung diseases [10]. While the contributions of IL-1 $\beta$ to several lung immune pathologies were identified more than a decade ago, experimental evidence has accumulated in recent years showing that other pro- and antiinflammatory members of the IL-1 family, including IL-33, IL18 and IL-37, also play pivotal roles in controlling these pathological processes. In this issue, Borthwick details the impact of this cytokine family on lung immunopathology, with special focus on lung fibrosis.

In summary, this issue of Seminars in Immunopathology will provide the readers with an overview of key processes controlling the immune homeostasis of the lung and the consequences of dysregulation of these processes, which leads to chronic inflammation, lung tissue injury and/or fibrosis. Great advances have been made in recent years in our understanding of the molecular mechanisms underlying immune-mediated lung pathologies due to the introduction of mouse models mimicking certain aspects of human disease. However, future translational work on primary patient material is required to validate these findings and to tackle the challenge posed by the growing impact of chronic and acute lung diseases on public health.

\section{References}

1. Fernandez-Botran R, Sanders VM, Mosmann TR, Vitetta ES (1988) Lymphokine-mediated regulation of the proliferative response of clones of T helper 1 and T helper 2 cells. J Exp Med 168:543-558

2. Mosmann TR, Cherwinski H, Bond MW, Giedlin MA, Coffman RL (1986) Two types of murine helper T cell clone. I. Definition according to profiles of lymphokine activities and secreted proteins. J Immunol 136:2348-2357

3. Hu W, Pasare C (2013) Location, location, location: tissue-specific regulation of immune responses. J Leukoc Biol 94:409-421. doi:10. 1189/jlb.0413207

4. Hussell T, Bell TJ (2014) Alveolar macrophages: plasticity in a tissuespecific context. Nat Rev Immunol 14:81-93. doi:10.1038/nri3600

5. Ginhoux F, Guilliams M (2016) Tissue-resident macrophage ontogeny and homeostasis. Immunity 44:439-449. doi:10.1016/j.immuni.2016. 02.024

6. Kopf M, Schneider C, Nobs SP (2015) The development and function of lung-resident macrophages and dendritic cells. Nat Immunol 16:36-44. doi:10.1038/ni.3052

7. Snelgrove RJ, Godlee A, Hussell T (2011) Airway immune homeostasis and implications for influenza-induced inflammation. Trends Immunol 32:328-334. doi:10.1016/j.it.2011.04.006

8. Licona-Limon P, Kim LK, Palm NW, Flavell RA (2013) TH2, allergy and group 2 innate lymphoid cells. Nat Immunol 14:536-542. doi:10. 1038/ni.2617

9. Cosio MG, Saetta M, Agusti A (2009) Immunologic aspects of chronic obstructive pulmonary disease. N Engl J Med 360:2445-2454. doi:10. 1056/NEJMra0804752

10. Barnes PJ (2008) The cytokine network in asthma and chronic obstructive pulmonary disease. J Clin Invest 118:3546-3556. doi:10.1172/JCI36130 